\title{
The EEG Correlates of Cerebellar Fits
}

\author{
Yannick Nadeau, Richard Desbiens
}

Can. J. Neurol. Sci. 2009; 36: 529-530

Originally described as "cerebellar seizures" by Wurffbain in $1691^{1}$, the term cerebellar fit is generally associated with the classical description of John Hughlings Jackson in $1871^{2}$ of a five-year-old boy with a cerebellar abscess and tetanus-like episodes. Cerebellar fits consist of headaches, alteration of consciousness, rigid extensor posturing of the extremities with stiffness of the trunk and eventually respiratory compromise and death $^{3}$. It is now known that cerebellar fits are associated with various entities, including tumor of the posterior fossa, encephalitis, acute hypoxia, herniation and any cause of sudden increase in intracranial pressure. The exact pathogenesis of these spells is still controversial: initially, cerebellar fits were considered an ictal phenomenon, but others suggested they represent decerebrate rigidity secondary to brainstem compression $^{4}$. Nonetheless, their presentation can easily be mistaken for epileptic seizures.

The electroencephalographic changes occurring during these episodes have only been described on a few occasions. We report on the electroencephalographic (EEG) correlates of cerebellar fits in one patient and provide a review of the literature.

\section{Case Report}

A 24-year-old woman was brought to the emergency department for a three-week history of paroxysmal headaches, worse in the morning, associated with nausea and vomiting.

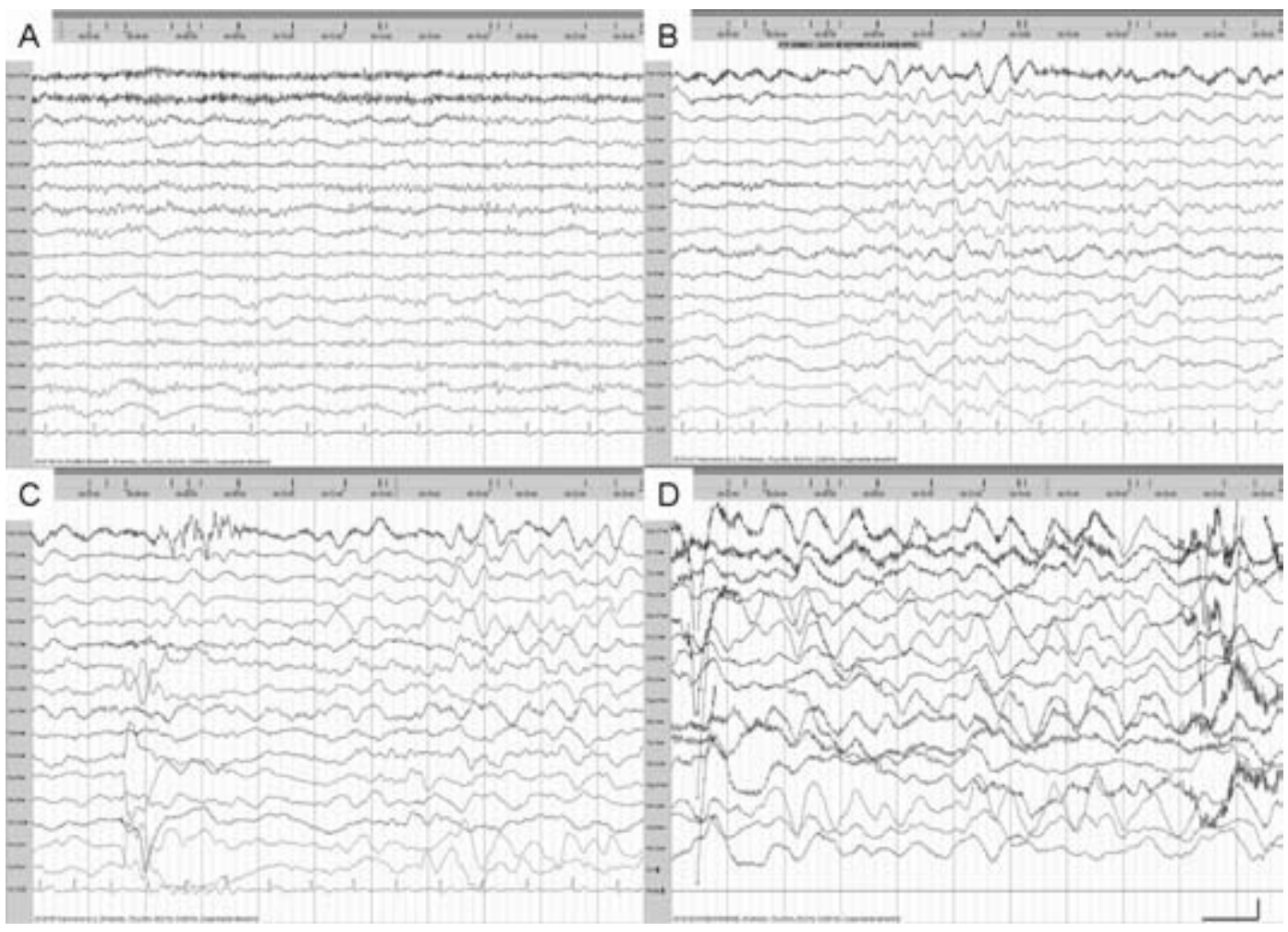

Figure 1: The EEG shows alpha rhythm (A). As the patient becomes unresponsive, bilateral disorganized polymorphic 1.5- to 5 Hz activity appears (B, $C$ and D). Calibration signal $1 \mathrm{sec}, 70 \mu \mathrm{V}(\mathrm{LFF} 0.5 \mathrm{~Hz}, \mathrm{HFF} 50 \mathrm{~Hz})$.

\footnotetext{
From CHAUQ Enfant-Jesus, Quebec, QC, Canada.

Received April 22, 2008. Final Revisions Submitted February 24, 2009

Correspondence to: Richard Desbiens, CHAUQ Enfant-Jesus, 1401 18th Street, Quebec, Quebec, G1J 1Z4, Canada.
} 
During the same period, she also experienced numerous falls, initially diagnosed as epileptic drop attacks. Her past medical history included Chiari I malformation, colpocephaly, dysgenesis of corpus callosum and hypoplasia of the anterior fossa. At age two, she underwent ventriculoperitoneal shunting for hydrocephaly. The patient was admitted for further assessments. While she was hospitalized, she presented multiple episodes of alteration of consciousness with rhythmic hyperextension of the limbs occurring every three to four seconds. The episodes lasted two minutes. Bilateral mydriasis and extensor plantar responses were noted during the episodes Consciousness typically returned within five minutes.

One episode occurred during a routine EEG. While in the tonic phase of the event, the EEG showed no rhythmic or epileptiform activity. Instead, the alpha rhythm (Figure 1A) was replaced by bilateral disorganized polymorphic slow activity (1.5-5 Hz) (Figures 1B, 1C and 1D). With resolution of clinical manifestations, the EEG spontaneously returned to normal within a few minutes, without any interictal epileptiform abnormality.

A brain MRI showed protrusion of cerebellar tonsils 15 millimetres below the foramen magnum with impaction of the bulbospinal junction (Figure 2). Disconnection of the shunt tubing in the neck was demonstrated on X-rays. A shunt revision procedure was performed under general anesthesia. An elevated CSF pressure was noted during surgery.

The episodes, diagnosed as cerebellar fits, disappeared completely after shunt revision and never recurred (follow-up of three years). The patient is on no antiepileptic medication.

\section{Discussion}

Cerebellar fits can easily be misdiagnosed as epileptic spells because they present with paroxysmal alteration of consciousness and abnormal stereotyped movements or postures. However, the underlying pathology and therapeutic approach are strikingly different. With modern imaging techniques, the diagnosis of intracranial hypertension secondary to tumor or bleeding is easy, but some aetiologies can still elude diagnostic imaging. The EEG is a useful, non-invasive, readily available diagnostic tool. In our patient, the diffuse polymorphic slow activity and absence of epileptiform abnormality, along with the clinical presentation, clearly oriented the investigation.

Information on the EEG changes associated with cerebellar fits is limited because there are only a few documented cases in the literature. In one published report, cerebellar fits in a patient with a pontocerebellar tumor were associated with a brief (seven seconds) period of suppression of electrical activity followed by diffuse high amplitude delta activity ${ }^{5}$. However, the recording was limited to only one side of the head and was terminated early because of disconnection of the electrodes due to head movements. In another report ${ }^{6}$, a patient suffered from one to ten-minute episodes of opisthotonic posturing with loss of consciousness caused by increased intracranial pressure following excision of a lumbosacral myelomeningocele. The EEG initially showed bilateral synchronous slowing before any clinical change, followed by diffuse flattening during the actual tonic posturing. Later during the event, generalized slow activity reappeared while consciousness was gradually regained. These rare recordings have a few features in common: non rhythmic,

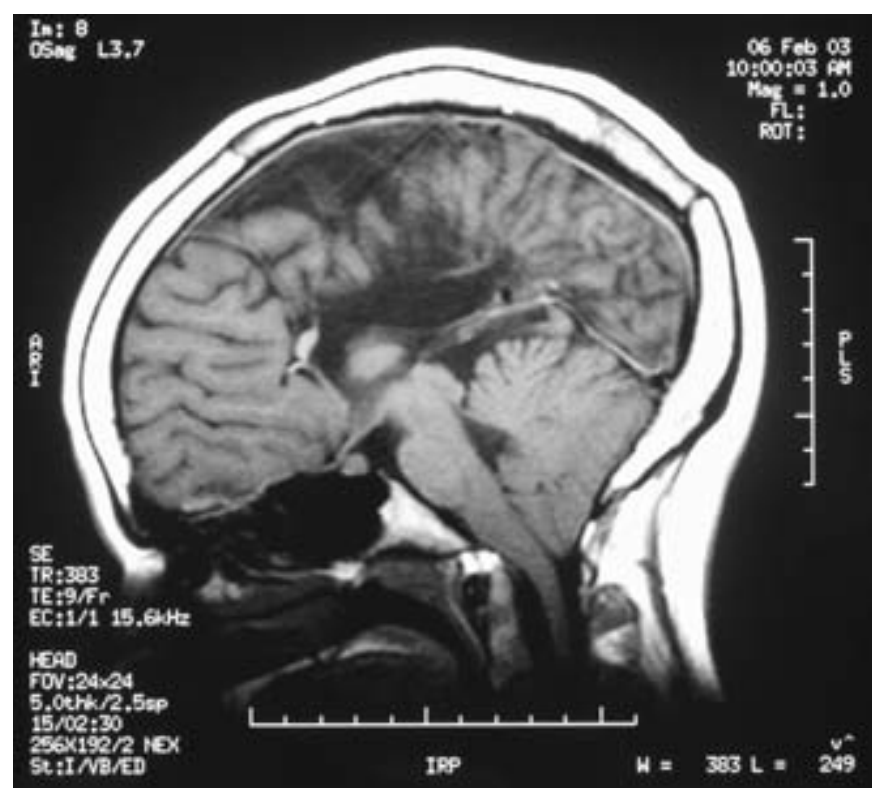

Figure 2: Sagittal T1WI MR showing dysgenesis of corpus callosum and protrusion of cerebellar tonsils 15 millimetres below the foramen magnum with impaction of the bulbospinal junction.

non periodic diffuse slow wave activity and loss of amplitude of EEG activity.

Cerebellar fits are associated with paroxysmal non epileptiform changes on EEG. In our patient, the electroencephalographic findings led to the right diagnosis and appropriate treatment of the potentially fatal underlying condition.

\section{Disclosure}

Richard Desbiens has received honoraria from UCB Pharma and Janssen-Ortho.

\section{REFERENCES}

1. McCrory PR, Bladin PF, Berkovic SF. The cerebellar seizures of Hughlings Jackson. Neurology. 1999;52:1888-90.

2. Jackson J. Case of tumor of the midline lobe of the cerebellum: rigidity in cerebellar attitude - occasional tetanus-like seizures. BMJ. 1871;242:528.

3. Penfield W, Jasper H. Epilepsy and the functional anatomy of the human brain. Boston: Little, Brown; 1954.

4. Wilson S. Decerebrate rigidity in man and the occurrence of tonic fits. Brain. 1920;43:220-68.

5. Gonsette R, André-Balisaux G, Colle J. Aspects électroencéphalographiques des crises toniques provoquées par une tumeur soustentorielle. Soc Electroenceph. 1959;101:290-3.

6. Strobos RRJ, Alexander Jr E. The electroencephalogram in cerebellar or tonic fits. EEG Clin Neurophysiol. 1960;12:491-4. 